CAHIERS DE

NARRATOLOGIE

\section{Cahiers de Narratologie}

Analyse et théorie narratives

$38 \mid 2020$

Lusor in Fabula. Jeu vidéo et nouvelles frontières du récit

\title{
Le serpent et le papillon. Temps et narration dans Life is Strange (DontNod, 2015)
}

\section{Tony Gheeraert}

\section{(2) OpenEdition}

\section{Journals}

Édition électronique

URL : https://journals.openedition.org/narratologie/11685

DOI : 10.4000/narratologie. 11685

ISSN : 1765-307X

Éditeur

LIRCES

Référence électronique

Tony Gheeraert, « Le serpent et le papillon. Temps et narration dans Life is Strange (DontNod, 2015) », Cahiers de Narratologie [En ligne], 38 | 2020, mis en ligne le 18 janvier 2021, consulté le 15 août 2022. URL : http://journals.openedition.org/narratologie/11685; DOI : https://doi.org/10.4000/narratologie. 11685

Ce document a été généré automatiquement le 15 août 2022.

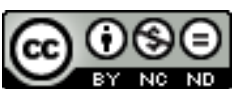

Creative Commons - Attribution - Pas d'Utilisation Commerciale - Pas de Modification 4.0 International - CC BY-NC-ND 4.0

https://creativecommons.org/licenses/by-nc-nd/4.0/ 


\title{
Le serpent et le papillon. Temps et narration dans Life is Strange (DontNod, 2015)
}

\author{
Tony Gheeraert
}

\section{"Le Temps est un enfant qui joue" \\ (Héraclite)}

Life is Strange, produit par DontNod et publié par Square Enix en 2015, se présente comme un récit interactif fondé sur une mécanique de choix à conséquences. Le joueur, qui incarne le personnage de Max, est amené à prendre divers types de décisions : certaines peuvent être anodines, d'ordre sentimental, ou, le plus souvent, engager des décisions éthiques très sérieuses, jusqu'au meurtre et à l'euthanasie. Le jeu se situe d'emblée sur le terrain de la délibération, et relève ainsi de la philosophie pratique. Le jeu présente une différence essentielle qui le distingue d'autres récits à embranchements : les développeurs ont pourvu la protagoniste du pouvoir de jouer avec le temps. Max peut l'arrêter, le rembobiner, ou même retourner dans un instant passé afin de le changer, pour peu qu'elle dispose d'une photo du moment concerné. S'il lui arrive d'abuser de son pouvoir, afin par exemple de devenir populaire dans son lycée ou de complaire à son amie Chloé, elle l'emploie aussi pour corriger des décisions prises par elle ou par d'autres, et dont les conséquences lui paraissent néfastes. De cette manière, ainsi que nous en prévient un intertitre au démarrage du jeu, les options prises par le joueur peuvent bouleverser non seulement le futur, mais le présent et le passé des personnages.

Life is Strange is a story based game that features player choice, the consequences

of al your in game actions and decisions will impact the past, present and future.

Choose wisely.-

En dotant l'héroïne de cette faculté inexpliquée, tout en la mettant aux prises avec des choix éthiques difficiles, Life is Strange manifeste le lien étroit entre temps et morale, souligné déjà par Jeanne Hersch: d'une part, en effet, selon la philosophe suisse, 
“l'exigence morale n'a de sens qu'au sein d'une situation temporelle engendrée par le passé et se développant vers l'avenir"'; et en même temps, l'impératif moral tend à un absolu hors du temps : la décision éthique obéit à un impératif catégorique qui "en s'arrachant à la relativité temporelle, a vocation à s'ériger dans l'éternité" ${ }^{2}$. C'est cette double dimension paradoxale du choix moral dans sa relation avec le temps qu'explore Life is Strange, à la faveur d'un scénario et d'une mécanique de jeu spécialement conçus en vue de cette finalité.

Le titre met en scène Max Caulfield et son amie d'enfance Chloé Price, à qui elle sauve d'abord la vie grâce à son pouvoir ; toutes deux mènent une enquête sur la disparition d'une élève, Rachel Amber. Leurs investigations révèlent la face noire du lycée, et confrontent Max à des situations difficiles: usage des drogues, handicap, relations abusives entre élèves et professeurs, sexualité et homosexualité, euthanasie, autant de sujets rarement abordés dans le jeu vidéo, et traités avec tact et finesse. Au terme de cinq jours, les héroïnes découvrent que Mark Jefferson, professeur de photographie séduisant et pervers, est l'auteur de l'enlèvement et de l'assassinat de Rachel. Pour perpétrer ses crimes, Jefferson a bénéficié de la complicité de Nathan Prescott, élève instable, héritier d'une lignée de grands bourgeois qui possèdent presque toute la ville. Parallèlement à l'enquête surviennent des événements étranges : éclipse inattendue, baleines échouées, oiseaux morts, et double lune. Max comprend in fine qu'en modifiant le temps, c'est elle qui a provoqué des dérèglements de plus en plus sérieux, jusqu'à déclencher une tornade susceptible de détruire la ville: en vertu du fameux effet papillon, selon lequel le battement d'aile d'un lépidoptère en Europe risque de provoquer une tempête au bout du monde, de légères modifications dans le tissu spatio-temporel peuvent entraîner des cataclysmes. Le choix final, inéluctable quelles que soient les options prises au cours du jeu, contraint l'héroïne à une douloureuse alternative : soit elle remonte une dernière fois dans le temps afin de sauver la ville, mais elle doit alors renoncer à user de ses pouvoirs, et par conséquent doit laisser périr Chloé; soit elle décide de sauver son amie, mais Arcadia Bay se trouve alors détruite sous ses yeux. La communauté des fans a désigné ce dilemme d'un jeu de mots : "Bay or Bae".

4 L'originalité du titre Life is Strange consiste dans la liaison étroite entre d'une part la mécanique ludique du choix moral à conséquence, et de l'autre le thème du temps qui structure à la fois le gameplay et l'ensemble du scénario. Tout système de choix à conséquence n'implique pas nécessairement une perspective morale : dans les livres dont vous êtes le héros des années 1980, les choix étaient guidés par l'intérêt, et visaient à l'efficacité pour survivre ou remporter la victoire. Dans bien des visual novels, c'est l'intérêt romantique qui guide les choix du joueur ou de la joueuse. Dans Life is strange, l'héroïne est confrontée à des tests qui l'engagent à se positionner certes parfois en vue de son propre bonheur et de son intérêt (par exemple être ou non suspendue du collège), mais le plus souvent pour ceux de son entourage. Là est d'ailleurs l'une des originalités les plus frappantes du titre : les épreuves morales auxquelles est confrontée la protagoniste n'influencent que modérément son sort personnel. Jusqu'au dernier acte, quelle que soit la conduite adoptée par l'héroöne, l'arc transformationnel visible en jeu reste peu impacté par les stratégies du joueur : c'est plutôt la réaction des autres 
personnages qui se trouve modifiée, ainsi que l'image de la protagoniste aux yeux du joueur lui-même.

5 Le game design et le système des personnages favorisent la superposition de trois temporalités qui correspondent à trois systèmes éthiques : l'urgence linéaire tragique, l'éternel retour cyclique d'inspiration nietzschéenne, et la spirale amérindienne. Ces trois conceptions de l'histoire et du temps contribuent bien sûr à multiplier les occasions de tension narrative; mais elles permettent aussi, plus profondément, d'opposer des valeurs morales et des choix d'existence dans ce jeu à caractère initiatique destiné d'abord à la jeunesse. Elles représentent trois manières d'être au monde: le réalisme magique de Life is Strange ${ }^{3}$ ne donne ainsi au joueur la possibilité de jouer avec le temps que pour faire miroiter ces possibles éthiques qui correspondent à différentes attitudes face à l'existence.

\section{Ligne : le game design comme destinée}

6 La première forme de temporalité est celle d'une linéarité dramatique et tragique ${ }^{4}$. Quelle que soit la liberté conférée au joueur face aux dilemmes qui se succèdent, Life is Strange se déroule selon une structure épisodique contraignante, pré-déterminée par les concepteurs. Une telle linéarité, qui progresse inéluctablement vers la scène du phare, s'apparente à celle de l'ancienne tragédie, dans un jeu qui réactive les questionnements caractéristiques de ce genre: libre-arbitre, responsabilité individuelle, poids des déterminismes. Sur le strict plan de la forme, la tension tragique repose sur la concentration de l'espace et du temps : l'histoire se déroule en un lieu unique, Arcadia Bay, coincé entre l'océan et la forêt, et dont le nom grec évoque non sans ironie le paradis originel mythique chanté par les poètes hellènes. L'intrigue est également resserrée dans le temps, puisque cinq jours suffisent à conduire à son terme le cours des événements. L'action, quoi que décide le joueur, progresse vers le final sur la falaise, théâtre d'une tornade surnaturelle qui menace de transformer pour de bon le paradis arcadien en enfer. Max avait prévu cette tempête, à laquelle elle avait assisté au cours d'un songe prémonitoire; or, ces rêves prophétiques sont fréquents dans la tragédie : ils révèlent que le temps est sous contrôle de forces obscures. Une sombre fatalité, perverse, tient entre ses mains tous les fils de l'histoire et s'attache à la perte des héros. À dix reprises, dans son journal, Max évoque son inévitable destinée : "How could this not be some kind of fate or destiny" ". La figure du destin hante le jeu jusque sur le T-Shirt du D.J. lors de la soirée du Vortex Club : "Fate".

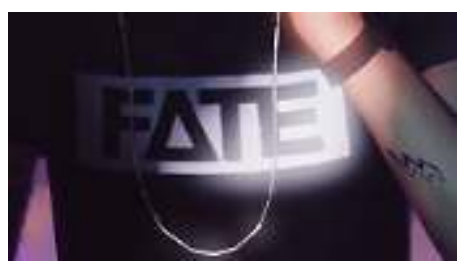

7 L'ombre du surnaturel plane sur la tranquille cité : “it's ominous" estime Max; le Tobanga, ou la biche mystérieuse dans le terrain vague contribuent à créer une atmosphère sacrée, que souligne encore le jardinier Samuel ; celui-ci, familier avec la magie indienne, développe longuement sa théorie du destin et de la fatalité, tout en laissant entendre à Max qu'elle est elle-même une shaman. 
Le moment tragique correspond toujours à un moment de désordre, une parenthèse de chaos où les lois habituelles du monde sont abolies: Oedipe-Roi se déroule pendant la peste de Thèbes, et dans Jules César, des météores inouïs traversent le ciel, écho céleste des drames contre-nature qui se jouent dans la cité des hommes. Il n'en va pas autrement dans Life is strange: chute de neige par beau temps, éclipse inattendue, et baleines échouées qui constituent, au cœur de l'intrigue, un moment de culmination; ces monstres marins symbolisent l'irruption du chaos, et la perturbation de l'harmonie au sein de ce paradis illusoire, révélant qu'il y a quelque chose de pourri au royaume d'Arcadia Bay. La contemplation de ces animaux morts, dans un décor crépusculaire d'une stupéfiante beauté, fascine l'apprentie photographe; mais le joueur ne peut s'empêcher de ressentir aussi un sentiment de révulsion face à ce drame morbide et inexplicable, symptôme d'un dérèglement cosmique ; le lendemain, la vision simultanée de deux astres lunaires suscite des angoisses d'apocalypse.

Dans cet univers déchu de toute certitude et de toute évidence morale, les personnages, sont mêlés, "ni tout à fait bons, ni tout à fait méchants" 6 : Dave, l'ancien policier devenu vigile, antipathique tuteur de Chloé, se livre à un sordide espionnage, mais ses intentions sont bonnes; l'odieux Jefferson en revanche, ravisseur pervers, tortionnaire et assassin, n'en est pas moins le séduisant Mentor dont le talent et la réputation ont attiré Maxine à Blackwell : avant de chercher à la tuer, le professeur de photographie dévoile à son élève les arcanes de son métier, et lui dispense de précieux conseils pour sa future carrière, à commencer par le plus essentiel et le plus ambigu : "Always take the shot". Nathan également, blouson doré capricieux et gâté, psychologiquement instable, est une victime autant qu'un bourreau: il inspire à la fois terreur et pitié, les deux émotions tragiques selon Aristote. Sans être criminelle, Max "passe du bonheur au malheur à la suite de quelque faute": son hamartia consiste non en une "perversité" naturelle, mais en une série d'erreurs de jugement ${ }^{7}$; elle a aussi fait preuve de démesure $\left(\right.$ hybris $\left.{ }^{8}\right)$ en jouant avec le temps et en voulant réécrire l'histoire. En termes de gameplay, l'ombre de la fatalité correspond à la linéarité d'un jeu dont toutes les étapes se déroulent de façon nécessaire et mènent au climax qui se déroule sur la falaise : le jeu est une machine infernale qui, pour parler comme Racine, "s'avance par degrés vers sa fin". L'une des spécificités du titre de Dontnod réside dans un pessimisme moral qui débouche sur la réactivation d'une antique conscience tragique, au point de nier le libre-arbitre sous couvert de laisser une pleine liberté au joueur. Fatalité et déterminismes viennent miner les chimères d'émancipation, dans la plus pure tradition grecque, shakespearienne, ou racinienne.

Quel que noir que puisse être l'univers tragique, il est éclairé par sa finalité cathartique. La tragédie, explique Aristote, tend en effet à un retour l'ordre qui soit aussi un retour au calme ${ }^{10}$. Dans Life is Strange, des deux dénouements possibles, celui de la mort de Chloé correspond le mieux aux exigences aristotéliciennes de la catharsis. Au déferlement des passions, et au chaos des forces déchaînées de la nature dans le dernier épisode, succède l'apaisement final au moment des funérailles solennelles de la jeune femme. 


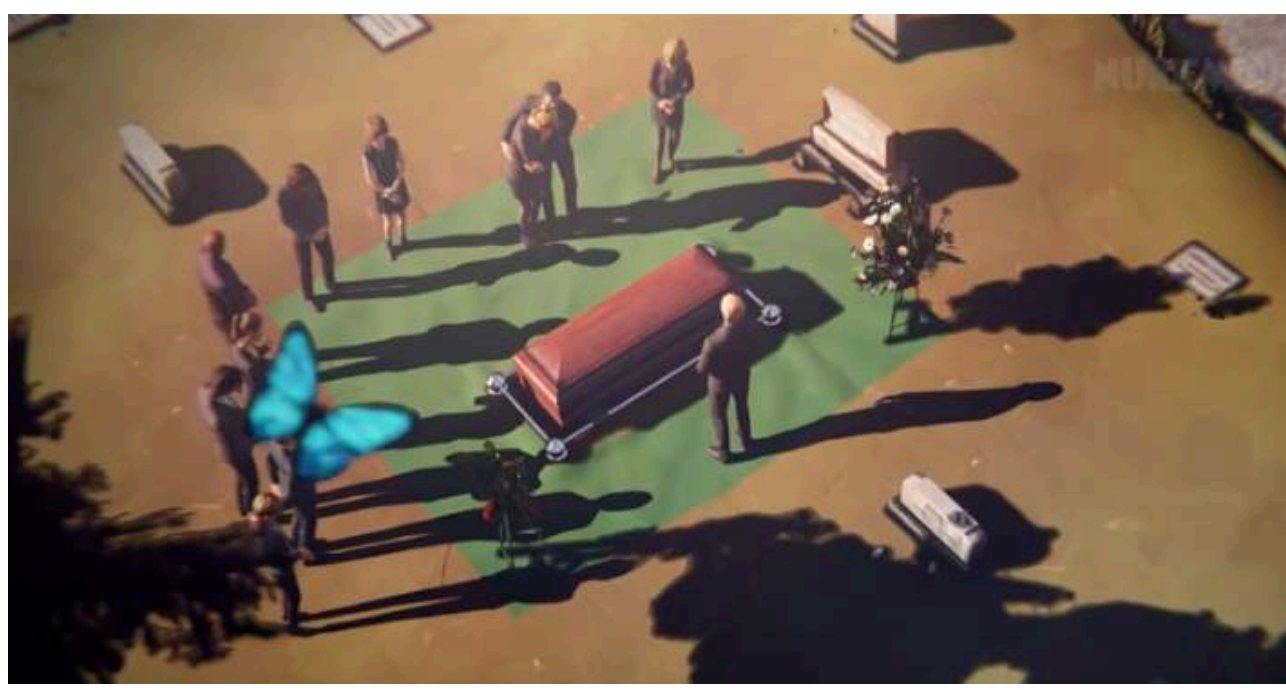

11 Le cercueil est ici entouré d'une communauté en deuil, réconciliée par le sacrifice expiatoire d'une Chloé christique, dont le nom résonne harmonieusement avec le cadre pastoral de la cérémonie funèbre : avatar de Déméter, celle dont le nom renvoie à la nature verdoyante périt pour que vivent sa mère et ses proches. La mort participe de l'harmonie nécessaire d'un monde qui unit les contraires. Le papillon qui volette sur le cercueil change de sens: il n'est plus le symbole du temps chaotique; il figure désormais l'acceptation de l'éphémère, et, s'il on se réfère au totémisme indien, à la transformation de Max, maintenant capable de passer à l'âge adulte. Comme celle d'Oedipe à Colone, la tombe de Chloé vient sceller la paix et promettre la possibilité du bonheur. C'est à l'effet de la catharsis qu'on peut attribuer la sérénité qui éclaire le visage Max à la vue du papillon, dans les toutes dernières images du jeu. La disparition acceptée de Chloé permet la restauration de l'ordre. La vie retrouve son équilibre. La tombe en Arcadie ne représente plus le scandale du trépas au Paradis : monument du souvenir, elle cimente l'unité de la communauté et garantit sa survie. Chloé la marginale trouve enfin, dans la mort, sa place dans cette ville qui l'avait rejetée.

Le joueur est-il invité à souscrire à ce triomphe du fatalisme et de la résignation, qui serait comme la "bonne fin" du jeu ? Rien n'est moins certain. Ce final est celui d'un insupportable renoncement: en annulant toutes les perturbations temporelles, Max admet que le monde ne peut et ne doit être changé. Il lui faut laisser Chloé périr assassinée dans les toilettes sordides de son lycée, et se soumettre à la loi du Destin, qu'on peut au mieux retarder, mais jamais contrarier. Telle était l'analyse de Chloé sur la falaise : "I think I should accept my fate... our fate [...] Maybe you've just been delaying my real destiny". Si Max accepte ce verdict et consent à la mort de son amie, elle acquiesce au Destin, et entérine du même coup un monde inamendable, où Victoria reste une peste, où Kate continue d'être harcelée, et où les Prescott poursuivront ces manigances immobilières qui finiront par engloutir le paradis arcadien. La fin désastreuse n'aura été que repoussée, et la catharsis n'aura été qu'une pause provisoire dans une marche à l'abîme qui finira malgré tout par la ruine de l'Arcadie.

\section{Cercle : retour du même et volonté de puissance}

"Bae or bay": l'autre dénouement, qui voit la destruction de la ville par la tornade, propose-t-il une meilleure issue au dilemme tragique ? La tempête possède-t-elle une 
valeur lustrale et purificatrice qui la rendrait désirable ? Chloé ne cesse pendant tout le jeu, jusqu'à l'épisode de la falaise, d'affirmer son libre-arbitre et de rejeter les déterminismes. Elle prend l'initiative, multiplie les transgressions, et rêve de quitter la ville avec son amie. Cette revendication de liberté et d'autonomie est représentée par le serpent qui se mord la queue sur son dernier T-shirt : l'Ouroboros, symbole d'origine orientale, mais aussi étroitement lié à la pensée de Nietzsche.

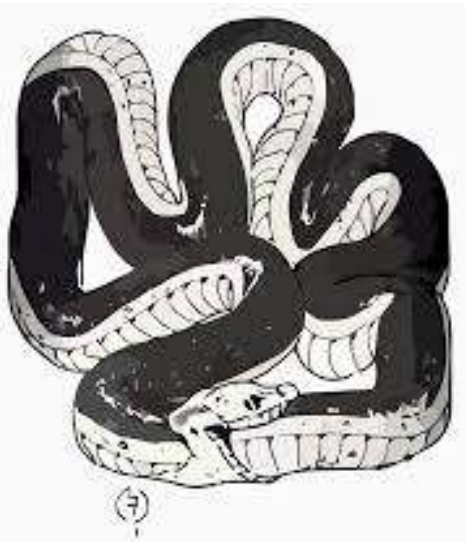

Associé à l'aigle, le serpent renvoie au mythe de l'éternel retour, central dans la pensée du philosophe allemand:

Je reviendrai, avec ce soleil et cette terre, avec cet aigle et ce serpent, - non pour une vie nouvelle, ou une meilleure vie, ou une vie ressemblante; - à jamais je reviendrai pour cette même et identique vie. (Ainsi parlait Zarathoustra; Troisième partie, « le convalescent », 1883)

Chloé, représentée au côté du "freedom eagle" sur un dessin de Max, compte parmi ses surnoms celui "d'Eagle-Eye" et porte un T-Shirt orné d'un aigle dans le prequel Before the storm : incarnant à la fois l'aigle et le serpent, elle apparaît ainsi comme une allégorie de l'Éternel Retour.

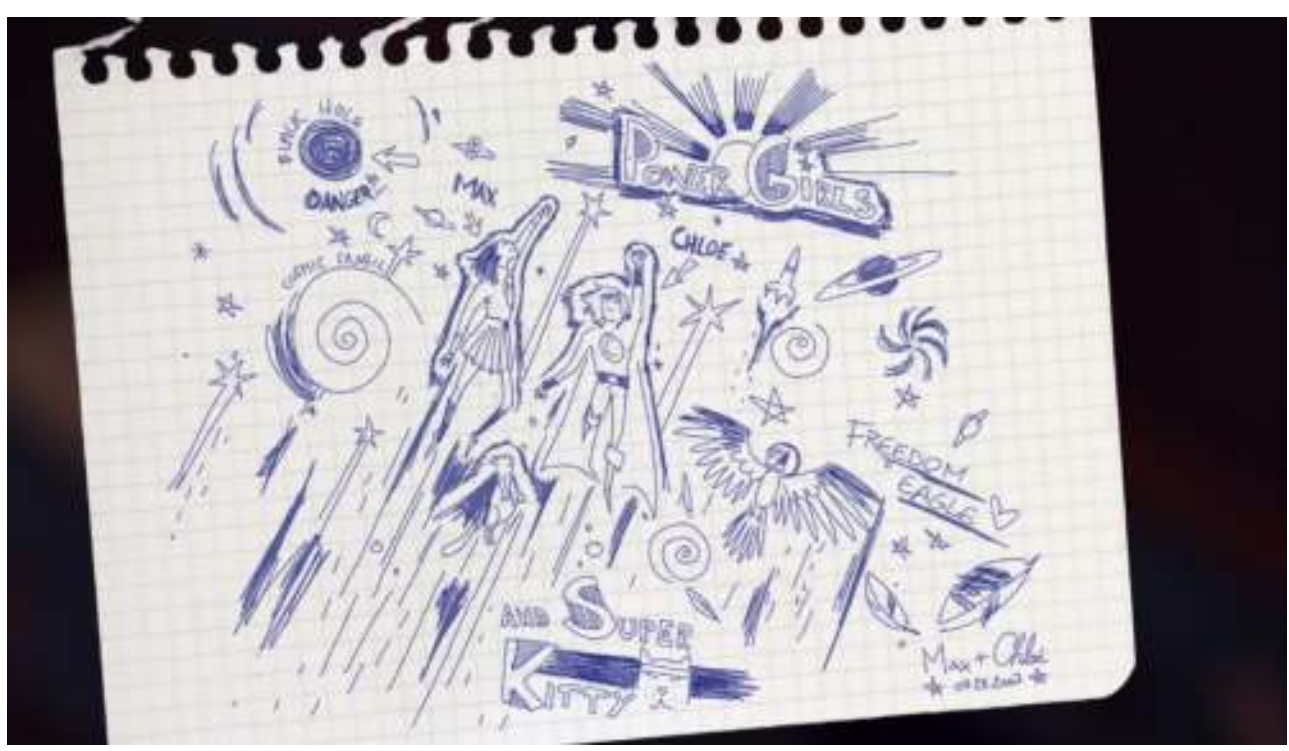

C'est encore sur le terrain du questionnement éthique que nous ramène en réalité ce concept temporel : chez Nietzsche, en effet, "l'Éternel Retour du même" est moins une 
théorie cosmologique ou ontologique qu'une hypothèse morale; le philosophe nous invite à vivre comme si notre existence était destinée à se répéter indéfiniment à l'identique :

"Et si un jour ou une nuit, un démon se glissait furtivement dans ta plus solitaire solitude et te disait : "Cette vie, telle que tu la vis et l'a vécue, il te faudra la vivre encore une fois et encore d'innombrables fois; et elle ne comportera rien de nouveau, au contraire, chaque douleur et chaque plaisir et chaque pensée et soupir et tout ce qu'il y a dans ta vie d'indiciblement petit et grand doit pour toi revenir, et tout suivant la même succession et le même enchaînement - et également cette araignée et ce clair de lune entre les arbres, et également cet instant et moi-même. Un éternel sablier de l'existence est sans cesse renversé, et toi avec lui, poussière des poussières!"11

17 L'Éternel Retour est une invitation à méditer sur la portée universelle de nos actes, comme s'ils devaient se reproduire à l'infini, mais il est aussi une affirmation de l'existence, un "grand oui à la vie", si l'on répond positivement à la question du démon. Ce concept du retour éternel du même met en valeur l'importance du moment présent, celui de la décision et de l'action, puisqu'il est appelé à se répéter à l'infini. L'affirmation joyeuse de l'éternel retour est la suprême leçon enseignée par Zarathoustra : si la vie est destinée à recommencer, il convient d'en vivre pleinement chaque instant. Il faut abandonner regret et remords, pour oser devenir ce que l'on est. Chloé, révoltée contre le conformisme moral petit-bourgeois, incarnation de l'indépendance, mérite à coup sûr d'arborer le T-Shirt à l'Ouroboros. Chloé, la rebelle, entraîne son amie dans ses rêves de transgression et d'évasion : pénétrer de nuit dans le lycée, ou partir à Los Angeles vivre avec elle une autre existence. Le système de jeu associe le joueur à cette quête : les nécessités de l'investigation, et la révolte de Chloé, conduisent les deux héroïnes à franchir les limites. Sortir en douce de Blackwell Academy, fouiller le bureau du proviseur ou prendre un bain de minuit dans la piscine, autant de manifestations d'un rêve d'émancipation que Chloé parvient à communiquer à Max. "Thelma et Louise" : c'est ainsi que Franck Bower interpelle le couple dans la décharge, préparant de loin l'un des deux dénouements, et révélant que le tandem de Life is Strange, entrainé par Chloé, partage avec le célèbre duo de Ridley Scott la même soif d'évasion. Le dénouement qui sauve Chloé peut ainsi être lu non seulement comme une ode à la liberté, mais un hymne à la Volonté de Puissance, dût-elle se bâtir sur une terre brûlée. Toutefois, le triomphe nietzschéen de la jeune femme au serpent et de son amie nous invite-t-il à prendre le parti de Chloé et à suivre la voie du Surhumain, au prix des ruines et des morts?

18 Le péan au Retour éternel et à la volonté de puissance viennent buter sur une image insupportable de ruines et de destruction. 


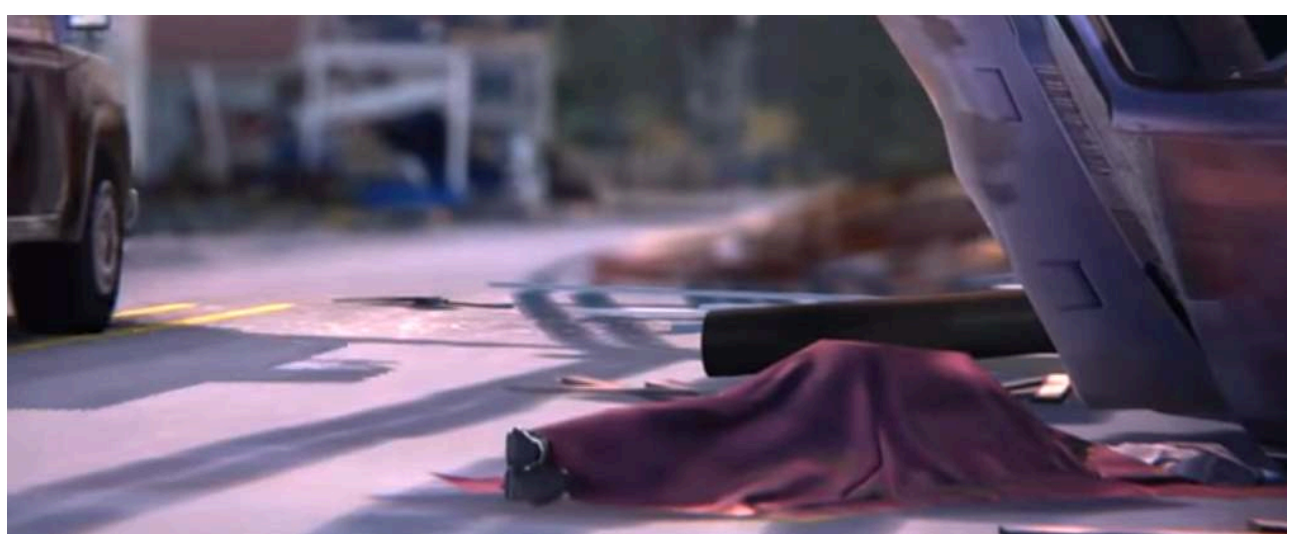

19 Cette fin égoïste laisse un goût d'insatisfaction et d'amertume au joueur, transformé in fine en simple spectateur d'une dernière cinématique. L'émancipation des protagonistes est aussi une fuite : elles hésitent brièvement après avoir dépassé un cadavre enveloppé d'une couverture, privé des funérailles que nous donnait à voir l'autre dénouement. Le van de Chloé s'arrête un instant, mais repart presque aussitôt. Les deux fugitives laissent les morts enterrer les morts. Comme le salut de Chloé Price, la liberté coûte un "prix" exorbitant : celui de l'ingratitude, de la solitude à deux, d'une asocialité qui fait régresser les héroïnes en-deçà des frontières de l'humanité. Dans ce décor contrenature, la sauvagerie l'emporte sur la civilisation, les défunts sont abandonnés, la cité dévastée est envahie par les bêtes fauves. La foi nietzschéenne dans l'affirmation de l'existence s'en trouve également ébranlée, si le prix à payer est si lourd.

L'Ouroboros manifeste l'affirmation d'une foi dans l'éternel retour qui est en même temps l'expression terrifiante d'une volonté de puissance: son triomphe implique la ruine d'Arcadia Bay. Assumer ses choix pour l'éternité, et comme s'ils devaient toujours recommencer, dussent-ils détruire le monde: telle est l'insoutenable leçon du dénouement "nietzschéen" et du temps circulaire.

Le titre de Dontnod tire sa profondeur de l'oscillation indécidable entre deux pôles contraires: la morne résignation d'un âge adulte assagi et apaisé, mais fataliste, et réduit à accepter la disparition de ses trésors les plus chers; et l'aspiration adolescente à une liberté sans bornes, dût-elle tout brûler derrière elle. La figuration de l'éternel retour prend également des résonances ironiques dans ce contexte de réitération temporelle: Chloé se réclame des valeurs nietzschéennes et du temps cyclique qui se répète à l'identique, alors qu'elle ne doit sa survie qu'aux distorsions spiralées introduites par son amie. A bien y regarder en effet, Chloé ne tire pas toutes les conséquences de l'Ouroboros. Bien au contraire: elle ne cesse de presser Max pour qu'elle use de son pouvoir et modifie le cours du temps, précipitant et aggravant ainsi la survenue du cataclysme. A l'éternel retour du même, elle préfère en réalité les jeux plus hasardeux de la spirale : une histoire qui se répète, mais non sans variations. Nous quittons ici le royaume circulaire de Chloé pour pénétrer dans celui de Max, domaine baroque des spires et des girations. 


\section{Spirale}

L'idée d'un temps spiralé est certes moins répandue que les conceptions cyclique ou linéaire, mais sans être complètement originale, puisqu'on la trouve dans certaines cultures amérindiennes, si essentielles par ailleurs dans le lore de Life is Strange ${ }^{12}$. Selon ces traditions, l'histoire se reproduit non tout à fait à l'identique, mais avec de subtiles variations : c'est ainsi que les Indiens Kiowa se représentent le cycle des saisons, qui se répètent certes, mais qui ne sont chaque fois ni tout à fait une autre, ni tout à fait la même.

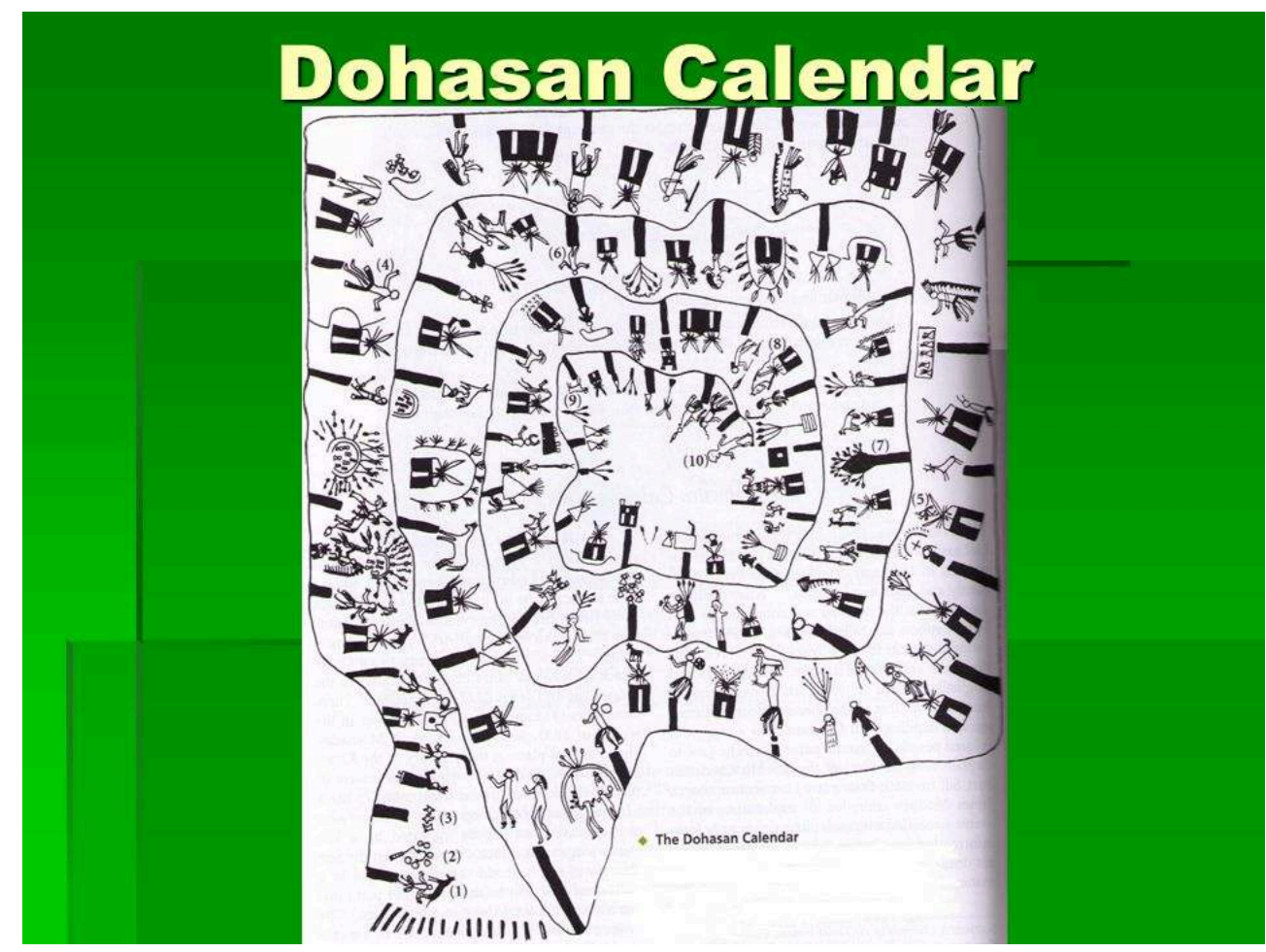

Calendrier Kiowa en spirale (1835) ${ }^{13}$

Mais dans Life is Strange c'est une fois de plus la perspective éthique et non cosmologique qui détermine le choix de cette figure : la spirale illustre la propriété de rembobiner le temps non pour qu'il se reproduise à l'identique, mais au contraire pour en changer le cours et évaluer les répercussions de ces modifications. La faculté magique dont dispose Max fonctionne en réalité comme une métaphore herméneutique des deux grandes éthiques contemporaines de la liberté : l'existentialisme et le conséquentialisme.

Je m'étendrai peu sur l'importance modélisatrice de la philosophie existentialiste dans Life is Strange: Luis de Miranda, chercheur à Edimbourg, a déjà proposé de rattacher le système de choix à la philosophie illustrée en France par Jean-Paul Sartre ${ }^{14}$. Cette doctrine postule que l'être humain est au départ une page blanche, dont l'identité ne se construit qu'au fil des décisions prises au cours de sa vie. Pour le philosophe français, la liberté, qui nous définit, se résume à nos choix, auxquels nous ne pouvons échapper et dont nous restons en toutes circonstances pleinement responsables. Non seulement nous avons toujours le choix, mais "nous sommes nos choix", estimait l'auteur de L'Etre et le néant. Chacune de nos décisions est libre, indépendante de notre passé, de nos 
résolutions précédentes, de notre situation, de nos déterminismes familiaux ou sociaux. Selon la formule fameuse, "l'existence précède l'essence", et ce n'est qu'à la mort, en définitive, que se fixe pour toujours notre être.

La mécanique de Life is strange, envisagée sous cet angle, s'accorde avec les présupposés que sous-tend cette vision du monde et de l'homme: Max n'existe qu'à travers ses prises de décision successives. L'arbre des cheminements narratifs n'est pas autre chose qu'un recensement de tous ses "chemins de la liberté", pour reprendre le titre d'une trilogie romanesque de Sartre. Max, comme les héros sartriens, est torturée par la difficulté des choix. La liberté, en effet, n'est jamais vécue par le joueur comme l'exaltation enivrante d'une toute-puissance; elle n'est pas griserie de l'illimité, et ne suscite pas ce sentiment de jouissance sans entrave que peut conférer un sandbox en monde ouvert. Le gameplay du choix à conséquence renvoie à une autre conception de la liberté, fermée, et conforme à la définition sartrienne : elle se limite à sélectionner une option possible parmi un ensemble de possibilités correspondant à des valeurs différentes. Les similitudes entre la mécanique de Life is Strange et la philosophie sartrienne confondent: dans L'existentialisme est un humanisme ${ }^{15}$, le philosophe prenait l'exemple d'un homme qui, pendant la guerre, se demandait s'il était plus moral de rester soigner sa vieille mère malade ou de s'engager dans la Résistance. Comme le remarque également Luis de Miranda, Sartre avait même imaginé, dans le roman Les Jeux sont faits ${ }^{16}$, le principe du rembobinage temporel: dans ce conte mi-amer miplaisant, des défunts pouvaient revenir à la vie et se voyaient offrir de changer certains des choix fondamentaux de leur existence. C'est ce type de liberté limitée et en vérité contraignante que met en scène Life is Strange. Le libre-arbitre ainsi entendu implique le poids d'une lourde responsabilité, et non l'ivresse d'un champ des possibles infini; il suscite l'angoisse, expliquent les existentialistes, du fait des conséquences vertigineuses qu'il entraîne, sans possibilité de rejeter sur un déterminisme ou une fatalité quelconque les désagréments éventuels qui suivraient nos décisions.

Le gameplay du jeu à conséquences permet sinon d'expérimenter tout à fait l'angoisse sartrienne, mais du moins de simuler ce vertige d'une liberté profondément anxiogène parce que bien trop lourde à porter. Cet existentialisme de nature morale n'est certes pas le propre de Life is Strange: les créations de TellTale ou de Quantic Dreams nous confrontent aussi à des situations délicates et comportant une dimension éthique, mais Life is Strange place son intrigue dans un univers réaliste, sans zombies, ni androïdes, ni créatures venues d'un autre monde : des jeunes filles d'aujourd'hui, en train de devenir des jeunes femmes, sont amenées à prendre position sur les grandes questions morales de leur époque, du harcèlement au suicide assisté. Cette dernière situation, qui se déroule dans un univers alternatif au milieu du jeu, préfigure le choix final : comme le héros de la conférence de Sartre, Max doit choisir entre sauver une personne qui lui est chère, ou la sacrifier pour le bien de la communauté. La scène du phare illustre la liberté existentialiste absolue : les orientations pour lesquelles Max a opté pendant le jeu ne viennent pas conditionner le choix final, qui reste indépendant des actions précédentes; le cours du jeu importe peu, Max pourra, in fine, librement choisir de sacrifier Chloé ou la ville, décision ultime qui l'essentialisera, si l'on peut dire, et en tout cas la figera pour toujours dans le souvenir du joueur ${ }^{17}$. Celui-ci doit cliquer : il n'y a pas d'échappatoire. Max ne peut s'abriter derrière aucune "mauvaise foi" (autre concept cher à Sartre) pour sortir de cette alternative. "Nous sommes embarqués", disait le Pascal du pari. "Nous sommes condamnés à être libres", renchérit Sartre aussi abruptement : Max a le choix, mais elle n'a pas celui de refuser de choisir, sans quoi le 
jeu s'arrête, puisque, dans ce premier opus, les créateurs avaient obstinément refusé la facilité du quick time event pour forcer l'intrigue à progresser ; le joueur, comme Max, a le temps, et peut longuement mûrir sa décision. Rien ne vient distraire le joueur de ce face-à-face avec lui-même sur lequel repose tout le gameplay ${ }^{18}$. Comme chez Sartre, la liberté devient dans Life is Strange le nouveau visage de l'antique Fatalité.

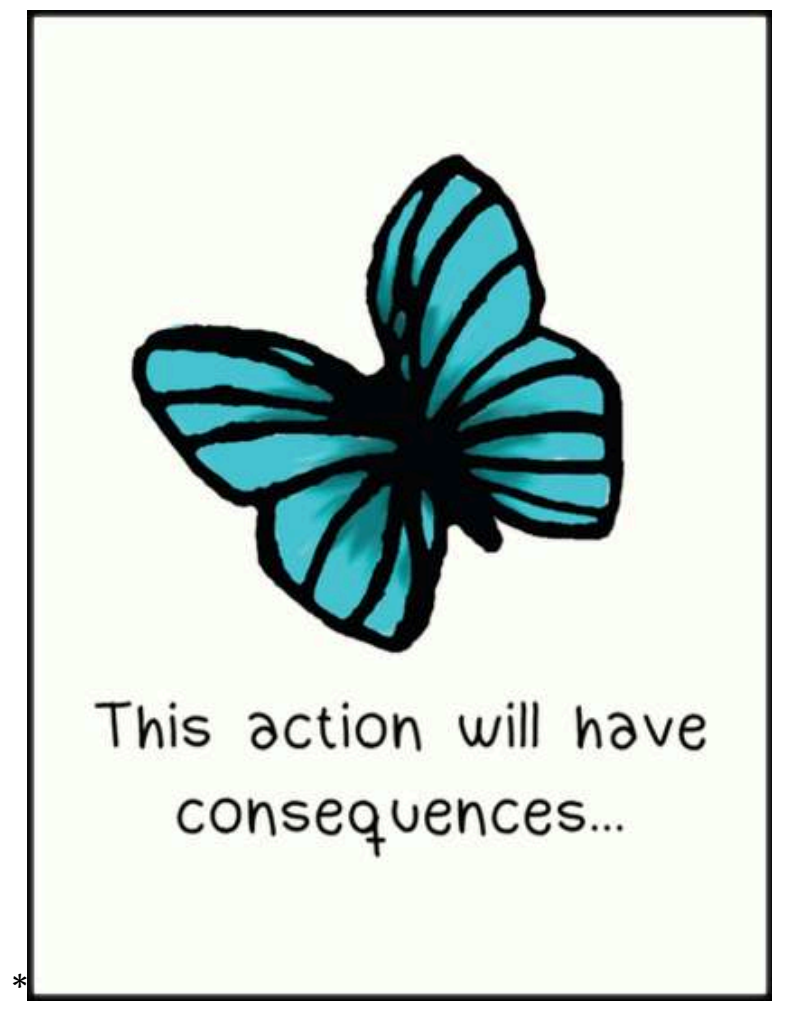

Plus encore peut-être que les contradictions d'un humanisme existentialiste qui reste très français et quelque peu daté, ce sont les questions ouvertes par l'utilitarisme et la philosophie analytique anglo-saxonne que soulève le temps spiralé de Max. La phrase récurrente qui ponctue le déroulement du jeu est à ce titre éloquente: "This action will have consequences".

Ce dernier terme renvoie à un courant très représenté dans les départements de philosophie outre-Manche et outre-Atlantique, appelé utilitarisme, mais parfois désigné également sous le nom de "conséquentialisme". Cette éthique téléologique affirme qu'un acte doit être jugé selon ses suites et non selon les intentions de celui qui l'accomplit, ni selon les qualités de l'acte lui-même. Pour les conséquentialistes, qui s'opposent ainsi à la vision déontologique kantienne, la valeur morale d'un acte ne découle que des avantages collectifs qu'il est susceptible de produire, et non de sa conformité à un bien ou une vertu préalablement identifiés et valables universellement. Le conséquentialisme, héritier des thèses de Jeremy Bentham (1748-1832), rencontre aujourd'hui un vif succès et influence nombre de comités d'éthiques et de décisions législatives. L'un de ses principaux promoteurs actuels est Peter Singer: il n'est pas indifférent que plusieurs des problèmes abordés par ce penseur américain soient traités dans Life is Strange, en particulier celui l'euthanasie volontaire. La situation dans laquelle se trouve Max, face à Chloé qui lui demande de 
mettre fin à ses jours, remplit très précisément les conditions exigées par Singer pour que se pose la question d'une légitimité éventuelle de l'euthanasie:

Voluntary euthanasia occurs only when, to the best of medical knowledge, a person is suffering from an incurable and painful or extremely distressing condition. In these circumstances one cannot say that to choose to die quickly is obviously irrational. ${ }^{19}$

Si le déontologisme traditionnel interdit le suicide assisté au nom d'un impératif catégorique de prohibition du meurtre ("tu ne tueras point"), les penseurs utilitaristes tiennent compte de la volonté du malade, et de la souffrance inutile que provoque une survie acquise par l'acharnement thérapeutique. Le choix insoutenable devant lequel se trouve placée Max, dans la réalité alternative où Chloé est une tétraplégique condamnée, propose au joueur de choisir entre un déontologisme strict et un conséquentialisme qui peut autoriser de donner la mort, dans ce cas précis d'un malade incurable et réclamant l'arrêt de ses souffrances. Le jeu, qui ne cesse de nous placer devant des dilemmes difficiles à trancher, relève ainsi de cette partie de l'éthique qu'on appelle la casuistique; cette science consiste en l'étude des cas de conscience où l'application des principes moraux généraux ne suffit pas à dégager une conduite. Le pouvoir dont Max a été dotée ne se résume pas seulement à intégrer à la narration une mécanique ludique éprouvée qui consiste à reprendre une progression au dernier check-point enregistré; il ne met pas non plus au centre du titre une quelconque interrogation sur le temps de type métaphysique ; il n'introduit pas même, dans ce jeu pourtant teinté de nostalgie, une méditation sur la fuite du temps; il ne repose pas non plus sur l'exploitation d'un de ces paradoxes temporels chers à la science-fiction. En réalité, la capacité rétrotemporelle de Max n'est qu'un support pour étudier des dilemmes moraux et leurs implications, dans une perspective philosophique conséquentialiste, puisque le joueur est sans cesse invité à se déterminer en fonction des suites prévisibles de ses décisions plus que de leur nature propre.

Le jeu, qui nous propose de choisir en fonction d'une évaluation des résultats attendus de nos actes, serait-il donc une apologie de l'utilitarisme conséquentialiste ? Certains détails nous invitent à en douter. En particulier, la présence du papillon dessiné à côté de la phrase récurrente constitue un démenti au conséquentialisme : l'animal symbolise dans le jeu la théorie du chaos et "l'effet papillon", autrement dit l'imprévisibilité des conséquences entraînées par nos actions, en raison de ce que les scientifiques appellent la sensibilité aux conditions initiales : nous ne pouvons maîtriser ni contrôler les suites de nos actes, qui peuvent être hors de proportion avec les faits qui les ont provoqués. Tout le jeu fonctionne en réalité comme une mise en garde destinée à nous alerter contre les dangers que représente l'utilitarisme: Max, en voulant corriger les conséquences de ses actes, ne parvient qu'à les aggraver, et à provoquer par surcroît une catastrophe météorologique entrainée par l'effet papillon que suscitent ses perturbations temporelles. L'éthique conséquentialiste, quelque séduisante et pragmatique qu'elle paraisse, ne saurait donc constituer un système valide pour conduire nos prises de décision. La mécanique du jeu à conséquence, le temps spiralé et l'effet papillon convergent pour dénoncer la limite fondamentale du conséquentialisme: la théorie du chaos rend toute anticipation impossible, et ruine ainsi le fondement de cette philosophie utilitariste qui suppose la possibilité de calculer précisément et rationnellement les répercussions de nos faits et gestes. Nul ne peut se déterminer selon les conséquences de ses actes car nul ne peut se flatter de les connaître, de les anticiper, encore moins de les maitriser. C'est dans cette mise en 
garde à caractère éthique que se rejoignent ainsi les différents aspects du jeu, et que gameplay et narration trouvent leur indissoluble cohérence.

C'est en particulier lors du choix final que le calcul des utilités perd toute forme de validité, et alors même que plus aucun doute ne subsiste sur les conséquences prédictibles de la décision. Le choix ultime a été préparé pendant tout le cours du jeu comme un piège inextricable, aux multiples facettes. Il peut être envisagé comme une variante extrêmement retorse d'un problème éthique classique en philosophie analytique anglo-saxonne : celui du tramway, formalisé pour la première fois en 1967 par Philippa Foot ${ }^{20}$.

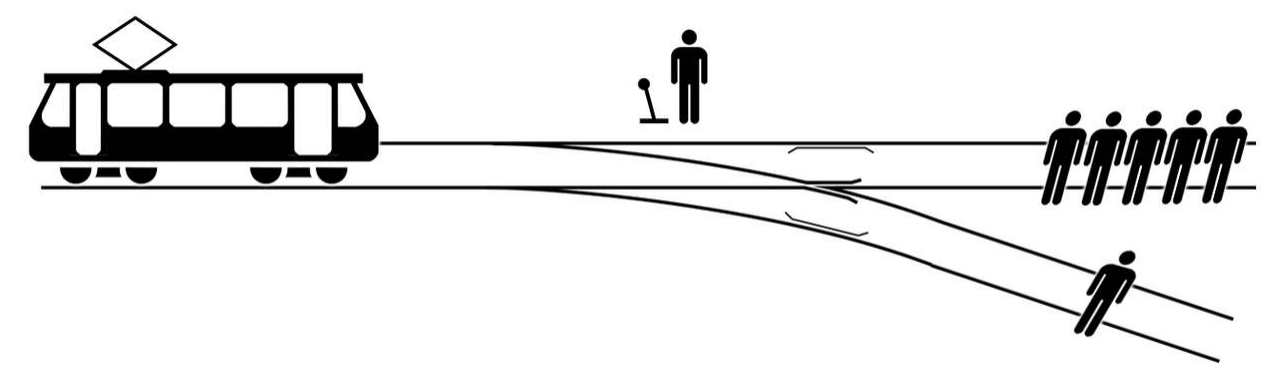

Dans sa formulation classique, il représente un tramway fou menaçant de tuer cinq hommes s'il poursuit sa route. La seule manière d'éviter ce drame consiste à actionner l'aiguillage, mais sur l'autre voie se trouve un individu qui périra alors nécessairement. Faut-il toucher au levier d'aiguillage, pour tuer avec certitude un homme, mais en sauver cinq autres ? Le problème a reçu beaucoup de variantes, et celle que nous propose Life is Strange est particulièrement redoutable. Dans la présente version, Max est à la fois le conducteur du train (puisqu'elle est responsable de la tempête) et l'agent d'aiguillage (puisqu'elle peut, en sacrifiant Chloé, sauver des milliers d'habitants). La solution déontologique consisterait pour Max à refuser d'user une fois de plus d'un pouvoir dont elle connaît maintenant la perversité ; mais le conséquentialisme, qui évalue un acte au regard du profit pour le plus grand nombre, lui recommande au contraire de sacrifier Chloé et d'user une dernière fois de la faculté rétrotemporelle : mais comment se résoudre à la cruauté de faire périr délibérément son amie la plus chère ? Dans Life is Strange, le problème du tramway a été spécialement construit de manière à n'admettre aucune solution, ni conséquentialiste, ni déontologique, et ainsi à faire basculer le joueur d'un cadre éthique normatif (quelle est la meilleure action possible ?) à une vision tragique impliquant le choix entre deux systèmes de valeurs à la fois valides et incompatibles.

Philippa Foot, n'inventa le paradoxe du tramway que pour montrer la vanité de ces calculs d'apothicaire en matière de morale, au profit d'un sentiment immédiat de ce qui constitue l'action bonne. Selon la philosophe, l'éthique des conséquences doit céder le pas à la vertu, et l'intuition morale doit l'emporter sur l'arithmétique. Entre déontologie et conséquentialisme, il existe une troisième voie, celle de l'éthique des vertus, selon laquelle c'est la qualité de la volonté qui détermine celle de l'acte. Comme l'explique R. Maggiori, pour Philippa Foot, "le point essentiel de la réflexion morale est de savoir quel genre de personne on est, et non quelles règles d'actions moralement correctes (d'utilité, de justice...) on doit suivre" ${ }^{21}$. Ainsi s'expliqueraient les opinions rassurantes données par Warren et Chloé à l'approche de la tempête : quelle que soit la décision de Max au moment crucial, elle sera la bonne, car elle aura été le produit d'une 
conscience pure et d'une intention droite. D'une âme vertueuse ne peuvent naître que des actes vertueux. L'ultime choix révèle ainsi la nature de la volonté qui a pris cette décision. C'est précisément sur cette révélation qui dépasse les frontières exiguës de la ratiocination casuistique que se termine Life is Strange: Max ne peut se contenter de mesurer les conséquences éventuelles de sa décision, aussi désastreuses d'un côté que de l'autre, puisqu'il n'existe aucun moyen rationnel de déterminer la bonne attitude qu'il conviendrait d'adopter. Ne subsiste qu'une morale intuitive du sentiment. Le jeu, comme la tragédie selon Aristote, culmine ainsi sur une anagnorisis, c'est-à-dire une reconnaissance, non pas dans le sein de l'action, mais de l'autre côté de l'écran: en choisissant de sacrifier son amie Chloé ou la ville d'Arcadia Bay, c'est la joueuse (ou le joueur) qui découvre les valeurs morales qui le guident, ses aspirations, et sa propre identité. Là où achoppent les systèmes des philosophes, là où la raison se trouve contrainte à l'abdication, triomphe une conception de la vertu fondée sur la conviction du for intérieur et de l'intimité de la conscience.

Si Life is Strange a été vécu avec tant d'intensité par le public depuis sa sortie, c'est parce que le joueur n'y est pas simple spectateur du désarroi tragique des héros : grâce à la vertu d'une mécanique ludique propre à faire tomber le quatrième mur, il est rendu en quelque manière responsable du choix final et de ses répercussions. Il devient lui aussi héros de cette tragédie de la liberté : il est embarqué. C'est par-delà le bien et le mal, dans l'horizon du tragique, que Max est amenée à se déterminer, et c'est aussi dans ce crépuscule moral que le joueur est invité à la rejoindre. Si les décisions n'ont finalement guère de prise sur le cours général d'une intrigue épisodique et linéaire, c'est sur le joueur qu'elles exercent leur influence; c'est lui qui se retrouve mis en cause, entraîné dans ce monde aux contours flous et aux principes incertains, emporté dans un tourbillon d'inquiétude et de flottement, et pour finir, amené à remettre en question ses propres choix en termes de morale et d'identité.

Life is Strange, jeu tout entier construit sur des questions éthiques, ne saurait toutefois se réduire à un manuel de cas à étudier et résoudre. Au contraire : la théorie du chaos vient réfuter tout moralisme facile et dogmatique, fondé sur des critères objectivables et calculables du vice et de la vertu. En authentique tragédie, le jeu se garde de délivrer une leçon. Il nous laisse méditer sur un tragique qui est au fond celui de la condition humaine, celui d'une vie au bord de la falaise. Life Is Strange rompt ainsi avec une tradition vidéoludique bien ancrée selon laquelle le terme de l'expérience devrait pouvoir se solder par un sentiment de victoire et de gratification symbolique obtenu à travers une "bonne" fin. Life is Strange n'est certes pas la première œuvre à suggérer la faillite des morales de la liberté et du calcul des conséquences : le thème est récurrent, tant dans les œuvres classiques que dans la culture populaire, de Sartre à Harry Potter et l'enfant maudit, en passant par le film intitulé L'Effet papillon auquel le jeu renvoie explicitement. Dans l'univers du jeu vidéo, le problème du tramway avait déjà été traité dans Fable III. Mais le gameplay du choix à conséquences, en brisant le quatrième mur, donne une dimension nouvelle à ce traitement esthétique des questions éthiques; il impose au joueur une responsabilité, voire une culpabilité, épargnées au spectateur de cinéma ou au lecteur de roman. Ceux-ci contemplent, certes avec effroi et pitié, mais de loin, les passions véhémentes dans lesquelles se débattent les protagonistes d'une tragédie. Désormais, le jeu vidéo permet au joueur d'entrer partiellement dans sa 
déstabilisante enceinte. De plus, dans Life is Strange, la profondeur psychologique et l'empathie des joueurs à l'égard non seulement de l'héroïne principale, mais de la plupart des habitants d'Arcadia Bay, transforme en drame humain le problème d'éthique formelle abstrait et désincarné posé par le dilemme du tramway. Chloé, Max, mais aussi Joyce ou Warren acquièrent, au fil des épisodes, une épaisseur au moins comparable à celle d'un héros de fiction dans un média traditionnel. C'est ce sentiment d'existence qui rend plus insurmontable le final du phare: nous ne sommes plus confrontés à un puzzle de logique formelle comme dans le trolley problem, mais à des êtres vivants face à des cas de conscience que ne saurait résoudre l'application stricte de critères objectifs. Face à l'impossibilité de prédire les conséquences de nos actes, et de dégager une conduite fondée en raison, ne reste que le sentiment, l'émotion, et surtout cette intime conviction, aussi indémontrable qu'irréfutable, d'avoir pris la bonne décision. Une morale du cœur, qui a ses raisons, mais que la raison ne connaît point.
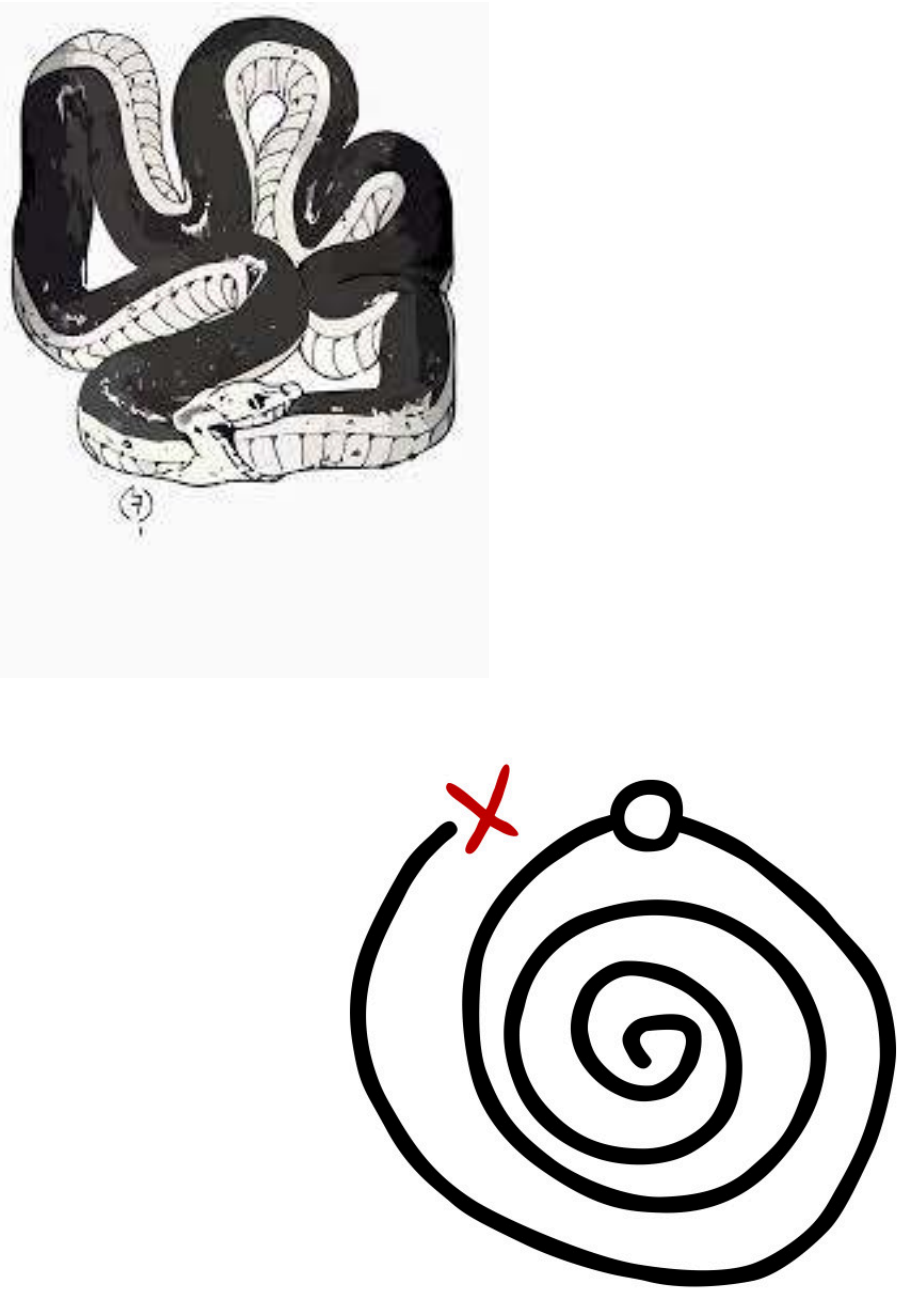


\section{NOTES}

1. Jeanne Hersch, "L'exigence morale aux prises avec le temps", Revue de Métaphysique et de Morale, 60e Année, No. 4 (octobre-décembre 1955), p. 413-416.

2. Ibid.

3. Voir sur ce point Christian Divine, "Life is Strange: the blue age of storytelling", Game Developers Conference Europe, 15-16 août 2016. En ligne: https://www.gdcvault.com/ play/1023848/-Life-is-Strange-The

4. Sur les liens étroits qui unissent la question du temps à celui de la tragédie, voir Gisèle Venet, Temps et vision tragique : Shakespeare et ses contemporains [1985], Presses de la Sorbonne Nouvelle, 2002, en particulier la question de la double temporalité dans Othello («Tragique et contingence »). En ligne : https://books.openedition.org/psn/4667

5. Journal de Max, Episode 5, vendredi 11 octobre 2013.

6. Jean Racine, Andromaque, première préface (1667).

7. Voir Aristote, La Poétique, chap. 13.

8. Aristote, La Rhétorique, II, 2.

9. Jean Racine, Britannicus, "préface" (1669).

10. Aristote, La Poétique, chap. 6: "La tragédie réalise une épuration (catharsis) de passions de ce type."

11. Friedrich Nietzsche, Le Gai Savoir, 1882, fr. 341.

12. "The calendar delivered by him to Scott is drawn with colored pencils on heavy manila paper, as is also the Sett'an calendar obtained by the author. In both, the pictographs are arranged in a continuous spiral, beginning in the lower right-hand corner near the center" (James Mooney, The Calendar History of Kiowa Indians, Seventeenth Annual Report of the Bureau of American Ethnology to the Secretary of the Smithsonian Institution, 1895-96, Government Printing Office, Washington, 1898, p. 143). Voir aussi Cheryl Wells, "Suns, Moons, Clocks, and Bells: Native Americans and Time. Cheryl Wells", conférence prononcée au département d'histoire de l'université du Wyoming en 2008.

13. Reproduit dans J. Mooney, op. cit., planche LXXV.

14. Luis de Miranda, "Life Is Strange and "Games Are Made': A Philosophical Interpretation of a Multiple-Choice Existential Simulator With Copilot Sartre", Games and Culture, vol. $13, \mathrm{n}^{\circ} 8, \mathrm{p} .825-842$.

15. Jean-Paul Sartre, L'Existentialisme est un humanisme [1946], Paris, Gallimard, FolioEssais, 1996.

16. Jean-Paul Sartre, Les Jeux sont faits, [1947], Paris, Gallimard, Folio, 2017.

17. Les développeurs ont choisi une autre voie pour Life is Strange II : la situation finale est au contraire largement conditionnée par les décisions prises par le joueur. La deuxième saison de Life is Strange prend ses distances avec l'existentialisme strict qui caractérisait le premier opus de la franchise.

18. https://gamergen.com/actualites/life-is-strange-dontnod-entertainment-squareenix-projet-feu-vert-260526-1 
19. Peter Singer, Practical Ethics, $2^{\text {nd }}$ edition, Cambridge, University Press, 1993, p. 200.

20. Philippa Foot, "The Problem of Abortion and the Doctrine of the Double Effect ", Virtues and Vices, Oxford, Basil Blackwell, 1978; première édition: Oxford Review, numéro 5, 1967, p. 1-7.

21. Robert Maggiori, “Phillippa Foot”, Libération, 5 mars 2014.

\section{RÉSUMÉS}

Life is strange met en scène une héroïne capable de bouleverser le cours du temps. Mais à bien y regarder, ce sont plusieurs temporalités que déroule le titre de Dontnod, d'où découlent plusieurs conceptions de l'existence, toutes trois tragiques à leur manière : le temps linéaire et oppressif de la tragédie classique, tendu nécessairement vers la scène du phare et le dilemme final, d'abord : ce tragique du fatalisme et de la résignation culmine avec la mort de Chloé acceptant son destin, suivie de la cinématique des funérailles, à valeur cathartique. Le temps cyclique de Chloé, ensuite : l'ouroboros, serpent qui se mord la queue, symbolise une temporalité circulaire, affirmation d'un éternel retour du même, et expression terrifiante d'une volonté de puissance qui triomphe dans la ruine d'Arcadia Bay. Assumer ses choix pour l'éternité, et comme s'ils devaient toujours recommencer, dussent-ils détruire le monde : telle est l'insoutenable leçon du dénouement "nietzschéen", et le prix à payer pour la survie de Chloé, quoi qu'il en coûte. Enfin, le temps spiralé de Max, qu'on peut rattacher à la culture amérindienne : le retour en arrière permet à l'héroïne de modifier le cours des événements, et donne au joueur l'illusion d'une contingence et d'une malléabilité que les événements du cinquième épisode démentiront. Au contraire de l'ouroboros, la spirale figure le temps du regret, du remords et du ressentiment, du "si seulement..." (if only revient sans cesse dans les propos et les pensées de l'héroïne). La spirale manifeste aussi un parti pris moral antithétique des principes nietzschéens, mais tout aussi douteux ou ambigus : l'affirmation que la valeur morale d'une décision dépendrait de ses conséquences éventuelles. Le jeu use de la mécanique ludique pour introduire un questionnement sur l'utilitarisme moral, en débat dans les départements de philosophie anglosaxons, pour en dénoncer les principes.

A travers le système du choix à conséquence qui permet partiellement de modifier le cours du jeu, tout en gardant jusqu'à la fin un fil directeur scénaristique unique, les créateurs mettent en scène l'articulation délicate de la contingence et de la nécessité. Les différentes temporalités renvoient à trois manières d'aborder le monde : l'abandon résigné et fataliste à la destinée ; le ressentiment et le refus d'endosser ses propres ses décisions; ou au contraire l'affirmation de ses choix, assumés jusqu'à l'insoutenable. Dans tous les cas, quoi qu'on choisisse, on ne sort de l'Arcadie de l'enfance que pour entrer dans l'enceinte invivable du tragique - le monde adulte.

\section{INDEX}

Mots-clés : Fiction interactive, narration environnementale, temps, tragique, déontologie, conséquentialisme 
AUTEUR

TONY GHEERAERT

Université de Rouen Normandie, CEREdI 